\title{
Role of Phacoemulsification versus Phacotrabeculectomy on IOP in Patients with Primary Narrow Angle Glaucoma
}

\author{
HESHAM I. EL-SOROGY, M.D.*; ASHRAF I. MOAWAD, M.D.*; ABDUL-MONEM EL-HESSY, M.D.*; \\ MONA ABD EL-KADER, M.D.* and MOHAMMED F. EL-ASHRI, M.Sc.** \\ The Departments of Ophthalmology, Faculty of Medicine, Mansoura* and Kafr El-Shiekh** Universities, \\ Mansoura \& Kafr El-Shiekh, Egypt
}

\begin{abstract}
Background: Cataract surgery alone has gained more acceptance as an alternative surgical approach for primary angle closure glaucoma management. This view was supported by the recent advances in phacoemulsification and intraocular lenses, which possess a greater safety and a quicker visual recovery, in addition to a substantial reduction of intraocular pressure, deepening of the anterior chamber, and widening the filtration angle.
\end{abstract}

Aim of Study: The current study is to evaluate the effects of phacoemulsification and Intraocular Lens (IOL) implantation versus phacotrabeculectomy on IOP in eyes with PACG and coexisting cataract.

Patients and Methods: The current study is a randomized control study that has been conducted on 40 patients who were diagnosed to have PACG and chronic narrow angle glaucoma. Patients were recruited and followed in the Department of Ophthalmology, Mansoura University and were divided into 2 groups; (Group A) patients who underwent phacoemulsification and IOL implantation, and (Group B) patients who underwent phacotrabeculectomy and IOL implantation.

Results: The preoperative mean IOP \pm SD for Group A was reduced from $24.76 \pm 9.87 \mathrm{mmHg}$ to a mean of $12.17 \pm 1.68$, $11.88 \pm 1.32,11.88 \pm 1.32$, and $11.88 \pm 1.32$ at 1 week, 1 month, 3 months, 6 months and 12 months respectively. A statistically significant reduction in the IOPs was recorded at all-time points $(p$-value $<0.05)$. Similarly, the preoperative mean IOP \pm SD for Group B was reduced from $24.56 \pm 7.19 \mathrm{mmHg}$ to a mean of $11.55 \pm 1.19,11.28 \pm 1.04,11.28 \pm 1.04$, and $11.28 \pm 1.04$ at 1 week, 1 month, 3 months, 6 months and 12 months respectively. A statistically significant reduction in the IOP were recorded at all time points $(p$-value $<0.05)$.

Conclusion: The results are collected and statistically analyzed as well as published. We found that the phacoemulsification with IOL implantation is associated with comparable results with the conventional surgery (phacoemulsification with IOL implantation and sub-scleral trabeculectomy) but the later is associated with higher incidence of complication.

Correspondence to: Dr. Mohammed F. El-Ashri, E-Mail: Profashri16@gmail.com
Key Words: Primary narrow angle glaucoma - Intraocular presuure - Phacoemulsification - Phacotrabeculectomy.

\section{Introduction}

ANTERIOR displacement of the iris-lens diaphragm causing a shallow anterior chamber remains the major predisposing factor in Primary Angleclosure Glaucoma (PACG) [1]. Eyes with angle closure is likely to have a shallow anterior chamber due to anteriorly displaced iris-lens diaphragm compared with normal eyes [1-3]. Prior studies reported that cataract surgery widened the anterior chamber angle in eyes with PACG [4]. Previous studies proved that primary lens extraction not only reverses the acute attack of PACG but also achieves long-term IOP control [5-7].

Cataract surgery alone has gained more acceptance as an alternative surgical approach for primary angle closure glaucoma management. This view was supported by the recent advances in phacoemulsification and intraocular lenses, which possess a greater safety and a quicker visual recovery, in addition to a substantial reduction of intraocular pressure, deepening of the anterior chamber, and widening the filtration angle. Glaucoma management is far from optimum. Glaucoma filteration surgery (trabeculectomy) with or without antifibrotic agents remains the mainstay of surgical treatment. However, the high complication rate and the unpredictable post-operative course and degree of Intraocular Pressure (IOP) reduction led many to consider other treatment options not dependent upon external filtration. Some of these options included improvement of internal filtration such as viscocanalostomy, canaloplasty, i-stent, drainage to suprachoroidal space (golden shunt), or decreasing aqueous production by endoscopic cyclophotocoagulation [8-10]. Another option 
emerged after observing a reduction of IOP after cataract surgery $[\mathbf{1 1 , 1 2 ]}$.

The progress in the technique and technology of phacoemulsification and Intraocular Lenses (IOL) made cataract surgery the most commonly performed procedure with highly predictable visual and intraocular pressure outcome [13]. These two factors (visual improvement and IOP reduction) encouraged some ophthalmologists to treat some types of glaucoma by phacoemulsification and IOL implantation alone aiming for either a definite treatment or a step in the management plan that can be safely followed by a future glaucoma surgical intervention when necessary [14].

\section{Patients and Methods}

The current study is a randomized control study that has been conducted on 40 patients who were diagnosed to have PACG and chronic narrow angle glaucoma. Patients were recruited and followed in the Department of Ophthalmology, Mansoura University from July 2014 to January 2016 and were divided into 2 groups;

- Group A: Patients who underwent phacoemulsification and IOL implantation.

- Group B: Patients who underwent phacotrabeculectomy and IOL implantation.

Informed consents were obtained from all participants after confidentiality were assured.

\section{General inclusion criteria included:}

- Any patients who were diagnosed with Primary Angle Closure Glaucoma (PACG).

- Chronic narrow angle and were able to perform tests reliably, and signed an informed consent.

General exclusion criteria included:

- Any patient with concurrent active eye diseases such as diabetic retinopathy.

- Central retinal vein occlusion.

- Congenital eye diseases.

- Corneal opacity.

- Pseudoexfoliation syndrome.

- Uveities, and.

- Previous occular trauma or.

- Previous intraocular surgery.

Pre-operative evaluation:

Patients were evaluated by:

- Taking detailed medical and ophthalmologic history at the baseline visit especially antiglaucoma medication. Topical carbonic anhydrase inhibitors (CAIs) and miotics discontinued 2 weeks before surgery.

- Patients' demographic data such as age, gender, occupation, and place of residency were recorded.

- As for any pre-surgical patient, a history of systemic diseases and medications used including anticoagulants were necessary. If the patient was hypertensive, blood pressure was to be properly controlled as well.

- The recruited patients underwent a complete ophthalmological examination including.

-Visual acuity assessment using Landolt's broken rings chart.

- Refraction assessment using autorefractometer.

- Best Corrected Visual Acuity (BCVA).

- Anterior segment evaluation using slit lamp biomicroscopy.

- Measurment of introcular pessure using goldman applanation tonometry.

- Posterior segment examination using indirect ophthalmoscopy.

- Gonioscopic examination by Goldmann goniolens.

- All IOP lowering medications used during preoperative visits were collected and recorded.

-A-B scan ultrasography was performed to exclude any posterior segment pathology.

- IOL power was calculated using IOL master.

Surgical procedure:

- Surgical procedure for phacoemulsification and IOL implantation group:

Patients underwent phacoemulsification aspiration and IOL implantation. All surgeries were performed under local akinesia and analgesia (posterior sub-tenon injection of xylocaine and marcaine).

- Briefly, after anesthesia and sterilization with betadine $5 \%$.

- A standard phacoemulsification procedure was performed through a clear corneal incision of $2.80 \mathrm{~mm}$.

- An additional two side punctures were made for chopper insertion and facilitate irrigation and aspiration.

- Continuous Curvilinear Capsulorrhexis (CCC)about 4-6mm-was made after injection of viscoelastic (visococohesive) materials. 
- Hydrodissection.

- The nucleus was managed using chop and stop technique (making primary grove follwed by cracking the nucleus using phaco-tip against chopper), the phaco one parameter (power: $40 \%$, vaccum $20 \mathrm{mmHg}$ and flow rate: $20 \mathrm{cc} / \mathrm{min}$ ) and then nucleus pieces were aspirated by phaco two (power $30 \%$, vaccum $250-300 \mathrm{mmHg}$, flow rate $20 \mathrm{cc} / \mathrm{min})$.

- The cortical remnants were cleared by irrigation and aspiration.

- A foldable acrylic IOL was implanted in the bag.

- The corneal incision and side ports were finally hydrosealed.

Surgical procedure for phaco-trabeculectomy and IOL implantation group:

- A limbal-based conjunctival flap, 5-6mm, was fashioned with a Westcott scissor then tenon's capsule was excised.

- A blade 15 was used to fashion a partial thickness scleral flap 3-4mm. around $1 / 2$ thickness of the sclera. To avoid premature anterior chamber entry, the surgeon stops before reaching the limbus as the scleral flap is continued into the peripheral cornea.

- A keratome was used to enter the anterior chamber creating a two-plane incision for the phacoemulsification.

- An additional two side punctures were made for chopper insertion and facilitate irrigation and aspiration.

- Continuous Curvilinear Capsulorrhexis (CCC)about 4-6mm was made after injection of viscoelastic (visoco-cohesive) materials.

- Hydrodissection.

- The nucleus was managed using chop and stop technique (making primary grove follwed by cracking the nucleus using phaco-tip against chopper), the phaco one parameter (power: $40 \%$, vaccum $20 \mathrm{mmHg}$ and flow rate: $20 \mathrm{cc} / \mathrm{min}$ ) and then nucleus pieces were aspirated by phaco two (power $30 \%$, vaccum $250-300 \mathrm{mmHg}$, flow rate $20 \mathrm{cc} / \mathrm{min})$.

- The cortical remnants were cleared by irrigation and aspiration.

-A foldable acrylic IOL was implanted in the bag.

- The corneal incision and side ports were finally hydrosealed.
- Once the cataract surgery was completed and the IOL (foldable acrylic) was implanted, the surgeon did not remove residual viscoelastic, to help maintain the globe.

- Sclerostomy was performed under the scleral flap.

- A jeweler's forceps and Vaness scissor were used to create a peripheral iridectomy.

- Once it was ensured there was no bleeding from the sclerostomy or iridectomy, the scleral flap was closed with two interrupted 10/0 nylon sutures.

- The anterior chamber was deepened with BSS through the paracentesis incision and the eye observed to ensure that the anterior chamber was deep, the eye not hard, and that there is some flow of fluid from beneath the flap.

- Conjunctiva was approximated tightly to its original position with interrupted $10 / 0$ nylon sutures.

- Again, the anterior chamber was formed and the wound was checked for any leaks and need for reinforcement.

- Sub-conjunctival injections of a steroid, cycloplejic were given in the inferior fornix.

\section{Post-operative evaluation:}

Patients were examined at day 1 , then at 1 week, 1 month, 6 months and 12 months after surgery. IOP is evaluated and analyzed. A broadspectrum antibiotic such as moxifloxacin was used for 10-14 days as prophylaxis against infection in addition to prednisolone acetate $1 \%$ drops initially four times a day for 1 week then steroid stopped gradually. Also cycloplejic drops was prescribed from day 1 post-operative 3 times daily for 1 week.

All intraoperative and post-operative complications were recorded.

Post-operative fundus exams were done to ensure that there are no choroidal effusions, especially if the surgeon noticed shallowing of the anterior chamber or hypotony.

Data collected throughout history, basic clinical examination, investigations, imaging results and outcome measures was coded, entered and analyzed using SBSS software program (Version 16). Variables were compared using paired $t$-test and a $p$ value was considered statistically significant if equal or less than 0.05 . 


\section{Ethical consideration:}

The study has been approved by the institutional review board at Mansoura Faculty of Medicine, Mansoura University.

\section{Statistical analysis:}

Continuous variables were expressed as mean \pm Standard Deviation (SD), and categorical data were represented by number (n) and percentage $(\%)$. Variables were compared using a paired $t$-test using SPSS. IOP at different time points was analyzed by repeated measure one-way ANOVA. All statistical assessments were 2-sided, and a $p$ level of 0.05 was considered statistically significant.

\section{Results}

\section{Baseline data:}

A total of 40 eyes were enrolled in the study and were diagnosed to have primary angle closure glaucoma. Of those, 20 patients with a mean age of $62 \pm 9$ years ( 8 males and 12 females) underwent phocemulasification with IOL implantation (Group A), and 20 patients with a mean age of $61 \pm 9$ years (5 males and 15 females) underwent phacotrabeculectomy with IOL implantation (Group B).

Systemic history revealed that; four (4) patients in (Group A) and one (1) patient in (Group B) have diabetes millets, seven (7) patients in (Group A) and three (3) patients in (Group B) have blood pressure hypertension, three (3) patients in (Group A) and one (1) patient in (Group B) have both previous systemic diseases.

Ocular history revealed that; five (5) patients in (Group A) and seven (7) patients in (Group B) have hyperopic refractive error, three (3) patients in (Group A) and two (2) patients in Group B have previous closed angle attacks, five patients in each groups have contra-lateral intra-ocular surgery for angle closure glaucoma.

\section{Intraocular pressure:}

The baseline mean of Intra-Ocular Pressure (IOP) for Group A was $24.76 \pm 9.87 \mathrm{mmHg}$ and for Group B was 24.56 \pm 7.19 .

The preoperative mean IOP \pm SD for Group A was reduced from $24.76 \pm 9.87 \mathrm{mmHg}$ to a mean of $12.17 \pm 1.68,11.88 \pm 1.32,11.88 \pm 1.32$, and $11.88 \pm$ 1.32 at $1 \mathrm{w}, 1,3,6$ and $12 \mathrm{~m}$ respectively. A statistically significant reduction in the IOPs was recorded at all-time points ( $p$-value $<0.05$ ). Similarly, the pre-operative mean IOP \pm SD for Group B was reduced from $24.56 \pm 7.19 \mathrm{mmHg}$ to a mean of
$11.55 \pm 1.19,11.28 \pm 1.04,11.28 \pm 1.04$, and $11.28 \pm$ 1.04 at $1 \mathrm{w}, 1,3,6$ and $12 \mathrm{~m}$ respectively. A statistically significant reduction in the IOP were recorded at all time points ( $p$-value <0.05).

\section{Complications:}

Complications were reported in $20 \%$ of the study eight (8) patients. Of those as following:

- 3 patients have developed postoperative hypotony in Group (B), all were associated with shallow AC due to hyper-filtration and managed medically by topical cycloplegic and compressive eye patching. Choroidal detachment had been excluded by clinical examination and observed frequently for one month. Detailed dilated fundus is examined all-over the visits to exclude choroidal detachment nor hypotonus maculopathy.

- 1 patient had post-operative blebitis in Group (B), managed by frequent topical antibiotic (moxiflocacin) and topical cycloplegic and observed frequently for one month and then weekly for further two months. All-over the visits, no any $\mathrm{AC}$ reaction nor posterior segment involvement.

- 2 patients have developed post-operative persistent corneal edema in Group (A). May be due to preoperative persistent elevation of IOP. The surgeries were uneventful. Corneal edema had been relieved by topical hypertonic drops (5\% saline) for 4 weeks, topical frequent prednisolone acetate which tapered gradually and cycloplegic drops.

- Two patients have developed intraoperative rupture posterior capsule in Group (A). Both required anterior vitrectomy, one of them reciceve anterior chamber IOL in the same session, and the other undergone for scleral fixation IOL in separate session.

Table (1): Demographic data.

\begin{tabular}{lcc}
\hline & $\begin{array}{c}\text { Group (A) } \\
\text { Phaco- } \\
\text { emulsification } \\
\text { group }\end{array}$ & $\begin{array}{c}\text { Group (B) } \\
\text { Phaco- } \\
\text { trabeculectomy } \\
\text { group }\end{array}$ \\
\hline Age & $62 \pm 9$ & $61 \pm 9$ \\
Gender M:F & $8: 12$ & $5: 15$ \\
History of systemic & & \\
history: & 4 & 1 \\
DM & 7 & 3 \\
HTN & 3 & 1 \\
Both & & \\
Ocular history: & 5 & 2 \\
Ref. error & 3 & 5 \\
Previous attacks & 5 & \\
Contralateral surgery & & \\
\hline
\end{tabular}


Table (2): Baseline and follow up IOP values in both groups of phacoemulsification (Group A) and Phacotrabeculectomy (Group B) groups.

\begin{tabular}{lll}
\hline $\begin{array}{l}\text { Intra Ocular } \\
\text { Pressure (IOP) }\end{array}$ & Group (A) & Group (B) \\
\hline Baseline IOP & $24.76 \pm 9.87$ & $24.56 \pm 7.19$ \\
Follow-up at week & $12.17 \pm 1.68(p<0.05)$ & $11.55 \pm 1.19(p<0.05)$ \\
Follow-up at 1 month & $11.88 \pm 1.32(p<0.05)$ & $11.24 \pm 1.04(p<0.05)$ \\
Follow-up at 3 month & $12.24 \pm 1.32(p<0.05)$ & $11.28 \pm 1.04(p<0.05)$ \\
Follow-up at 6 month & $11.6 \pm 1.32(p<0.05)$ & $11.28 \pm 1.04(p<0.05)$ \\
Follow-up at 12 month & $11.6 \pm 1.32(p<0.05)$ & $11.28 \pm 1.04(p<0.05)$ \\
\hline
\end{tabular}

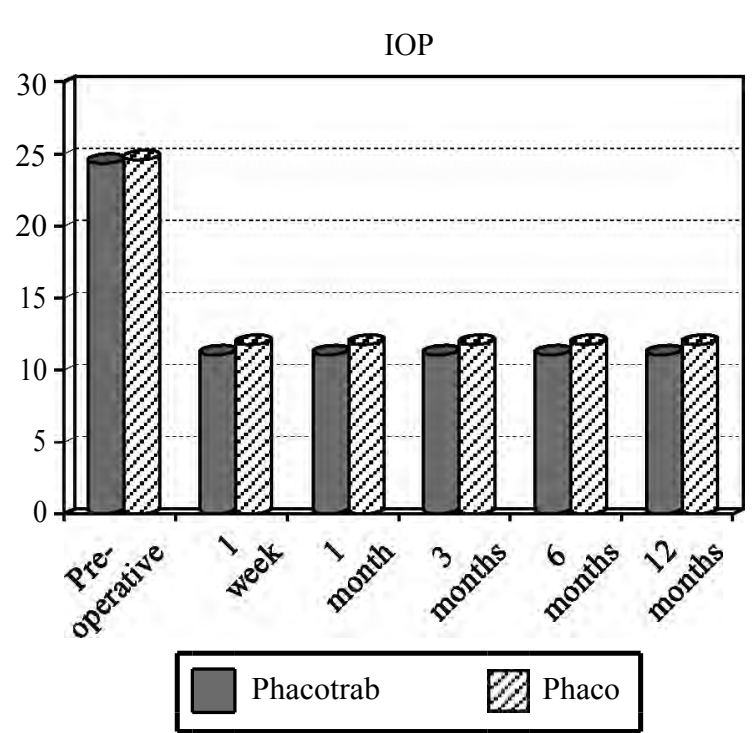

Fig. (1): Comparison between the IOP of the group treated with phacotrabeculectomy versus phacoemulsification in treatment of PACG. The results show no statistically significant difference between two groups.

\section{Discussion}

Surgical decision for management of coexisting glaucoma and cataract is challenging and needs to clarify many points such as; how glaucoma is appropriately medically controlled, progression rate, the degree of visual field loss, post-surgical visual outcome and, how safe and effective would be the decision to perform phacoemulsification or combined surgery for optimal management.

The glaucomatous eye may have miotic pupil, shallow Anterior Chamber (AC), zonular instability which would increase the surgical risks during cataract surgery. Also, an eye with previous glaucoma operation is more susceptible to increased intraoperative and postoperative surgical complications with subsequent cataract surgery. On the other hand, cataract extraction or subsequent YAG laser capsulotomy may compromise a successfully controlled glaucoma.

Coexisting cataract and glaucoma could be treated with different strategies including glaucoma surgery first, cataract surgery first/subsequent glaucoma surgery, or combined glaucoma and cataract surgery [15]. Increased the severity of glaucoma (advanced, medically uncontrolled disease, or rapidly progressive) may prioritize surgical managament for glaucoma. A maximum reduction in IOP can be established without pressure spikes and without added intraocular or subconjunctival alterations (like; anti-metabolites, valves or ologen) that may limit the success of filtering operations. Moreover, trabeculectomy surgery showed a better effect in phakic eyes than in aphakic or pseudophakic eyes. On the other hand, glaucoma surgery may be associated with increased progression of cataract with low patient's satisfaction, and may increase the risk of surgical complications with future cataract surgery. Moreover, cataract surgery may compromise a successful bleb and IOP control [16]. In fact, the benefit of successful filtration surgery in phakic eyes might be temporary and may be compromised with subsequent cataract surgery.

Current study includes total of 40 patients were enrolled in the study and were diagnosed to have primary angle closure or narrow angle glaucoma. Of those, 20 patients with a mean age of $62 \pm 9$ years ( 8 males and 12 females) underwent phocemulasification with IOL implantation (Group A), and 20 patients with a mean age of $61 \pm 9$ years (5 males and 15 females) underwent phacotrabeculectomy with IOL implantation (Group B).

In the current study, although it was noticeable that both groups have achieved the desired effect of statistically significant improvement in IOP during all time points, it was clear that combined surgery was associated with higher rates of complications.

In the present study, we compared results of phacoemulsification and phacotrabeculectomy surgery on eyes with PACG and significant cataract. We found that phacotrabeculectomy reduced the mean IOP from a preoperative level of $24.56 \pm 7.19$ $\mathrm{mmHg}$ to $11.28 \pm 1.04 \mathrm{~mm} \mathrm{Hg}$ at 12 months followup $(p<0.05)$, which was similar to patients treated with phacoemulsification surgery which was reduced from a preoperative level of $24.76 \pm 9.87$ $\mathrm{mmHg}$ to a post-operative level of $11.88 \pm 1.32$ $\mathrm{mmHg}(p<0.05)$ at 12 months follow-up. Nevertheless, there were no statistically significant differences between both groups, which is in agreement with the previous studies reported literature conducted by Clement CY et al., for 24 months follow-up [19]. The reduction in IOP in both groups was not only significant but also sustained; Fang 
Wang et al., proved the same results in his metaanalysis in 2015 [16].

Combined phacotrabeculectomy has been compared to cataract surgery alone for treating PACG in eyes with IOPs that were both controlled and uncontrolled $[\mathbf{1 9 , 2 0 ]}$. In a previous study, phacoemulsification alone showed a good control of IOP in both groups as well as the number of medications required for IOP control. In the medically uncontrolled glaucoma eyes, phacoemulsification alone reduced the IOP by nearly $8 \mathrm{mmHg}$ with, and the effect lasted until 2 years follow-up [21]. Control of IOP as defined in the study was achieved in more than $90 \%$ of patients in the two groups. The phacotrabeculectomy group showed higher rates of complications than for cataract surgery alone. Three patients had post-operative hypotony, and one patient developed blebitis.

It was concluded that phacoemulsification alone could be used as a surgical alternative to combined phacotrabeculectomy regardless if the IOP before surgery is controlled or not [22]. Although performing trabeculectomy achieved a better control on IOP, some investigators preferred phacoemulsification alone because of the decreased complication rate. These studies found that $14.8 \%$ and $2.9 \%$ of patients with controlled and uncontrolled IOP ultimately required trabeculectomy.

In conclusion, the decision for surgical management of cataract and glaucoma or considering cataract surgery for glaucoma treatment should be made based upon patient and disease characteristics and surgeons' preferences or skills. For coexisting glaucoma and cataract, there is an individual recommendation for every patient. Combined phacotrabeculectomy, glaucoma surgery alone or cataract surgery alone is performed when either a visually significant cataract or advanced uncontrolled glaucoma is dominating the scene. Conversely, criteria are not yet well defined for visual acuity or IOP level at which combined surgery is indicated. Generally, a moderate to severe medically uncontrolled glaucoma with visually significant cataract are good candidates. The multiplicity and advances of anti-glaucoma medications along with glaucoma laser treatment and the less favorable visual regain with glaucoma operations maybe in favor of primary cataract surgery in eyes with mild to moderate medically controlled glaucoma.

Cataract extraction in glaucoma patients who have non-visually significant cataract is found to be associated with modest reduction in IOP, which is not sustained, less predictable, and with tendency to return to baseline, especially in primary open angle glaucoma patients. In eyes with primary angle closure glaucoma, primary lens extraction restores the anatomy of anterior chamber and the angle, which eliminate an important factor in the pathogenesis of angle closure glaucoma in addition to its IOP lowering effect. These factors are in favor of primary cataract extraction than glaucoma incisional surgery in mild to moderate, medically controlled PACG eyes with visually significant cataract. Primary cataract extraction may have a role in uncontrolled mild to moderate PACG eyes with non-synechial angle closure.

\section{Conclusion:}

Cataract extraction has been shown to be valuable management plan in patients with PACG. In the past, cataract surgery recommendation alone in primary angle-closure patients who have uncontrolled IOP even in the presence of a visually significant cataract would have been controversial. Recently, avoiding a trabeculectomy in those patients is a recommended practice. The existing debate is over what to do for patients with uncontrolled IOP and nearly clear lenses and also what degree of angle closure is necessary for lens removal to have a therapeutic effect. That said, cataract extraction alone should be recommended for any angle-closure patient with a visually significant cataract and should be seriously considered as an alternative to filteration surgery in patients with uncontrolled IOP.

\section{References}

1- LOWE R.F.: Aetiology of the anatomical basis for primary angle-closure glaucoma. Biometrical comparisons between normal eyes and eyes with primary angle-closure glaucoma. Br. J. Ophthalmol., 1970; 54 (3): 161-169. http:// . www.pubmedcentral.nih.gov/articlerender.fcgi?artid=1 207665\&tool=pmcentrez\&rendertype $=$ abstract. Accessed December 13, 2016.

2- ALSBIRK P.H. : Anterior chamber depth and primary angle-closure glaucoma. I. An epidemiologic study in Greenland Eskimos. Acta Ophthalmol. 1975; 53 (1): 89104. http://www.ncbi.nlm.nih.gov/pubmed/1172916 . Accessed December 13, 2016.

3- LAN Y.W., HSIEH J.W. and HUNG P.T.: Ocular biometry in acute and chronic angle-closure glaucoma. Ophthalmologica, 221 (6): 388-94, 2007.

4- NONAKA A., KONDO T., KIKUCHI M., et al.: Angle widening and alteration of ciliary process configuration after cataract surgery for primary angle closure. Ophthalmology, 113 (3): 437-41, 2006.

5- GUNNING F.P. and GREVE E.L.: Lens extraction for uncontrolled angle-closure glaucoma: long-term followup. J. Cataract. Refract. Surg. 1998; 24 (10): 1347-56. 
http://www.ncbi.nlm.nih.gov/pubmed/9795850. Accessed December 13, 2016.

6- JACOBI P.C., DIETLEIN T.S., LÜKE C., ENGELS B. and KRIEGLSTEIN G.K.: Primary phacoemulsification and intraocular lens implantation for acute angle-closure glaucoma. Ophthalmology. 2002; 109 (9):1597-603. http:// . www.ncbi.nlm.nih.gov/pubmed/12208704. Accessed December 13, 2016

7- IMAIZUMI M., TAKAKI Y. and YAMASHITA H.: Phacoemulsification and intraocular lens implantation for acute angle closure not treated or previously treated by laser iridotomy. J. Cataract. Refract. Surg., 32 (1): 85-90, 2006.

8- YALVAC I.S., SAHIN M., EKSIOGLU U., MIDњLIOGLU I.K., ASLAN B.S. and DUMAN S.: Primary viscocanalostomy versus trabeculectomy for primary open-angle glaucoma: Three-year prospective randomized clinical trial. J. Cataract. Refract. Surg., 30 (10): 2050-7, 2004.

9- SPIEGEL D. and KOBUCH K.: Trabecular meshwork bypass tube shunt: Initial case series. Br J Ophthalmol. 2002; 86 (11): 1228-31. http://www.pubmedcentral.nih. gov/articlerender.fcgi?artid=1 771358 \&tool=pmcentrez \&rendertype=abstract. Accessed December 13, 2016.

10- MINCKLER D.S., BAERVELDT G., ALFARO M.R. and FRANCIS B.A.: Clinical results with the Trabectome for treatment of open-angle glaucoma. Ophthalmology, 112 (6): $962-7,2005$

11- WISHART P.K. and ATKINSON P.L.: Extracapsular cataract extraction and posterior chamber lens implantation in patients with primary chronic angle-closure glaucoma: Effect on intraocular pressure control. Eye (Lond), 3 (Pt 6): 706-12, 1989

12- GUNNING F.P. and GREVE E.L.: Uncontrolled primary angle closure glaucoma: Results of early intercapsular cataract extraction and posterior chamber lens implantation. Int Ophthalmol. 1991; 15 (4): 237-47. http://www ncbi.nlm.nih.gov/pubmed/1917318. Accessed December 13, 2016.

13- RESNIKOFF S., PASCOLINI D., ETYA'ALE D., et al.: Global data on visual impairment in the year 2002. Bull World Health Organ, 82 (11): 844-51, 2004.

14- WANG Y., SHENG Y., WANG M. and TAO J.: Management of Different Subtypes of Primary Angle Closure with Phacoemulsification and Viscogoniosynechialysis. Semin. Ophthalmol., May 2016: 1-5.

15- KURIMOTO Y., PARK M., SAKAUE H. and KONDO T.: Changes in the Anterior Chamber Configuration After Small-Incision Cataract Surgery With Posterior Chamber Intraocular Lens Implantation. Am. J. Ophthalmol., 124 (6): 775-80, 1997.

16- WANG F. and WU Z.H.: Phacoemulsification versus combined phacotrabeculectomy in the treatment of primary angle-closure glaucoma with cataract: A Meta-analysis. Int. J. Ophthalmol., 9 (4): 597-603, 2016.

17- EID T.M.: Primary lens extraction for glaucoma management: A review article. Saudi J. Ophthalmol., 25 (4): $337-$ 45, 2011.

18- WETZEL W.: Ocular aqueous humor dynamics after photodisruptive laser surgery procedures. Ophthalmic. Surg., 25 (5): 298-302, 1994.

19- REBOLLEDA G. and MUÑOZ-NEGRETE F.J.: Phacoemulsification in eyes with functioning filtering blebs: A prospective study. Ophthalmology, 109 (12): 2248-55, 2002

20- MOGHIMI S. and LIN S.: Role of phacoemulsification in angle closure glaucoma. Eye Sci., 26 (3): 121-31, 2011.

21- THAM C.C.Y., KWONG Y.Y.Y., LEUNG D.Y.L., et al.: Phacoemulsification versus combined phacotrabeculectomy in medically controlled chronic angle closure glaucoma with cataract. Ophthalmology, 115 (12): 2167-73.e2, 2008. 


\section{دور إستحلاب عدسة العين \\ فى مناجزة حالات المياه الزرقاء الآوليّة ضيقدة الزاوية}

المياه الزرقاء هى مرض العصب البصرى ويسبب تغيرات مطردة فى العصب البصرى ويتبع ذلك تغيرات مقابلة فى مجال الآبار. تعتبر

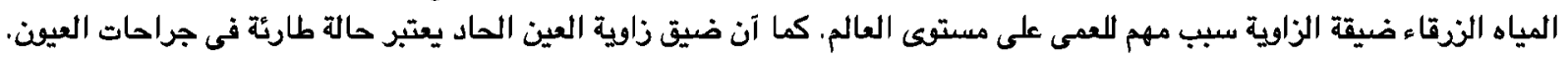

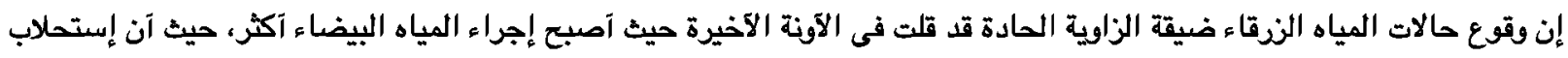

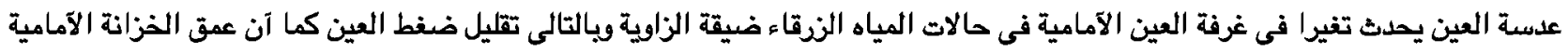

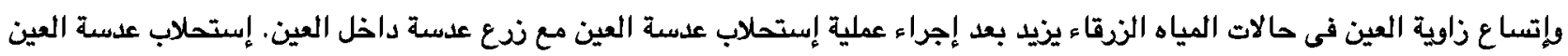

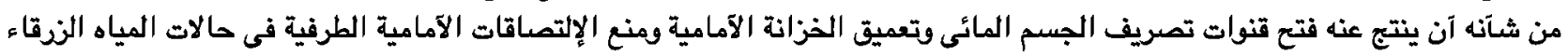

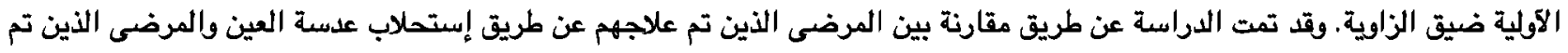

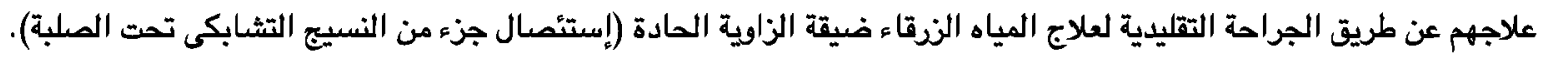

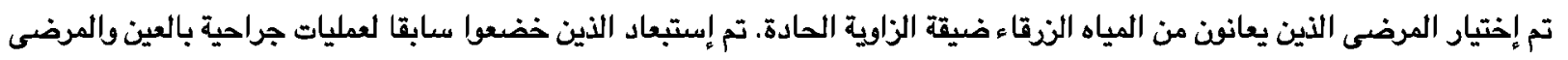

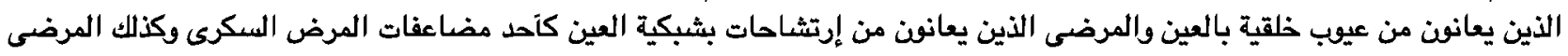

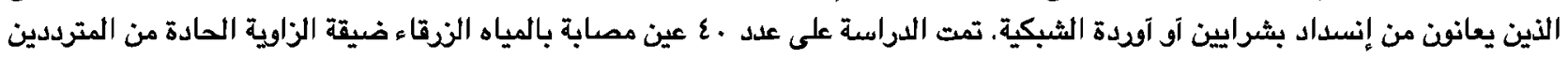

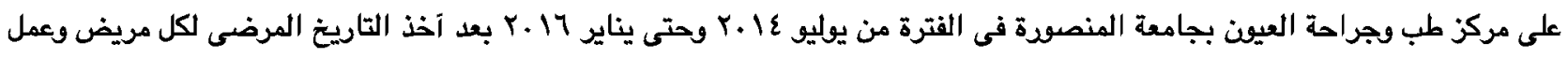

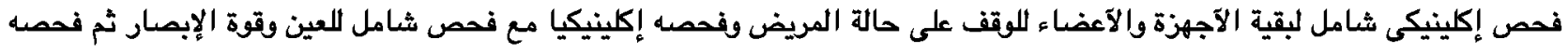

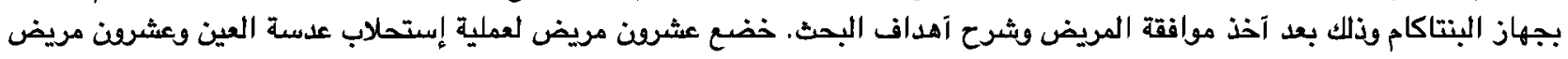

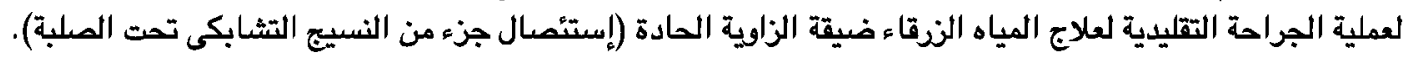

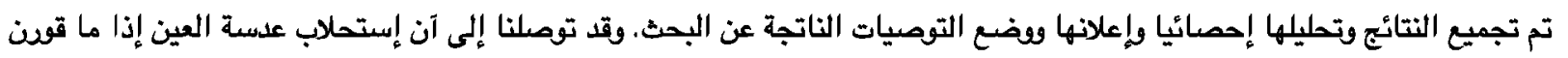

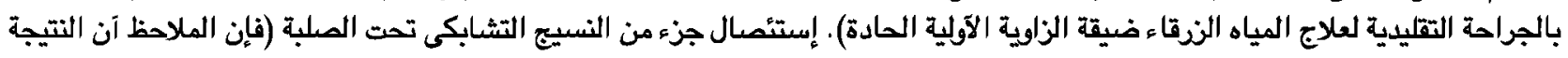

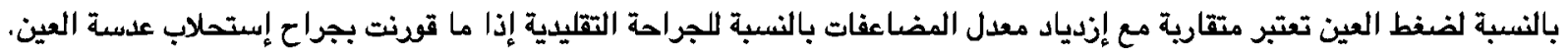

